\title{
A NEW TYPE OF SEMI-INSULATING MATERIALS
}

\author{
R.P. LEON AND E.R. WEBER \\ Department of Materials Science, University of California, Berkeley, CA 94720, USA \\ and \\ Materials Science Division, Lawrence Berkeley Laboratory, Berkeley, CA 94720, USA
}

The introduction of $\mathrm{Cu}$ in $\operatorname{In} \mathrm{P}$ at $700^{\circ} \mathrm{C}$ and higher temperatures results in both initially $p$-type and $n$-type $\mathrm{InP}$ become semi-insulating. It is also observed that thermally stable In-Cu rich precipitates form, that the concentration of deep levels is negligible and that InP:Cu samples exhibit inhomogeneities and anomalous transport behavior. The buried Schottky barrier model is the only model thus far studied which is consistent with these experimental observations. This model has general applicability, and its possible relevance to other semiconductors is examined. The conditions necessary for forming quasi-intrinsic semiconductors by metallic precipitation are discussed.

PACS numbers: 72.80.Ey

Obtaining semi-insulating semiconductors is possible when a large enough concentration of metallic precipitates exist in a crystal so that the space charge regions which form when a metal and semiconductor are in intimate contact could overlap and deplete the sample of free carriers. This situation would have the effect of making the semiconductor intrinsic.

This model was initially proposed [1] to explain the behavior of GaAs grown at low temperatures by molecular beam epitaxy (MBE) [2] and then annealed. In this material, a lower than normal growth temperature permits introduction of a large amount of excess arsenic. This excess arsenic is responsible for the creation of a large concentration of antisite defects $\left(\mathrm{As}_{\mathrm{Ga}}\right)$, but upon annealing, the majority of the excess As forms precipitates, creating a high concentration of hexagonal As clusters [3]. Annealed low-temperature MBE GaAs thus exhibits semi-insulating behavior and also has a large concentration of As precipitates. The situation in this material is still controversial, due to the presence of a large number of $\mathrm{As}_{\mathrm{Ga}}$ defects which are at least partly responsible for pinning the Fermi level near midgap. Also, epitaxial material is more difficult to analyze and electrical measurements are quite laborious.

More recently, it has been observed $[4,5]$ that $\mathrm{Cu}$ diffusion in InP greatly increased its resistivity, creating semi-insulating (SI) material with diffusion temperatures as low as $700^{\circ} \mathrm{C}$. Both $p$ - and $n$-type samples become semi-insulating with $\mathrm{Cu}$ doping, but midgap levels could not be found in sufficient concentrations to explain the reduction in carrier concentration by compensation from deep donors 
or acceptors. Furthermore, anomalous transport behavior was observed in a very drastic reduction of both electron and hole mobilities, and evidence was found for the existence of pockets of highly conducting material from both magnetic and electrical measurements. This inhomogeneous conductivity and the presence of a large concentration of $\mathrm{Cu}-\mathrm{In}$ precipitates [6] is very compatible with the buried Schottky barrier model, where regions of conductive material exist in between depletion zones when the concentration of precipitates is not sufficiently large or uniform. The study of InP:Cu has the added advantage of ease. in certain measurements that can be best accomplished in bulk rather than epitaxial material. The fact that $\mathrm{Cu}$ has a very small substitutional solubility in InP makes compensation by deep levels almost negligible in this material, therefore the effect of precipitates alone can be best understood.

Besides the two materials discussed above, the buried Schottky barrier model might be responsible for the electrical properties observed in GaP:Cu, GaAs:Li and fast neutron irradiated $\mathrm{GaAs}, \mathrm{CdTe}, \mathrm{CdS}$ and $\mathrm{Ge}$. In $\mathrm{GaP}: \mathrm{Cu}$ [7] it was found that both initially $n$-type and $p$-type GaP greatly increased in resistivity, but the concentration of deep donors was orders of magnitude below what would be required to explain this change by compensation mechanisms. Recently, it has been observed that Li doped GaAs [8] attains resistivities in the $10^{7} \Omega \mathrm{cm}$ range regardless of the type before diffusion. In the 1960's and 1970's, evidence for metallic clusters (spikes) was observed in fast neutron irradiated materials [9]. This metallic spike model could explain the observed changes in infrared absorption and electrical behavior. A reduction in carrier concentration was also present in this case, even though the role of point defects cannot be neglected in heavily neutron irradiated samples.

Determining if metallic precipitates play a role in the properties of any semiconductor is quite relevant, and the following discussion will address some of the appropriate characterization techniques, as well as the conditions required for this mechanism to form technologically applicable semi-insulating materials.

The observation of a decrease in carrier concentration in both initially $n$-type and $p$-type materials is usually a good indicator that buried Schottky barriers are responsible for this increase in resitivity. This phenomenon is observed in $\mathrm{InP}: \mathrm{Cu}$, $\mathrm{GaP}: \mathrm{Cu}[7]$ and GaAs:Li [8].

In all these cases, deep levels were not found in sufficient concentration to account for the observed transition to semi-insulating behavior. Figure 1 shows a plot of carrier concentration as a function of inverse temperature for InP:Cu samples that were originally $n$-type (nominally undoped). This plot shows the decrease in electron concentration as a function of $\mathrm{Cu}$ diffusion temperature. Figure 2 shows similar plots for samples that were originally $p$-type and that also became semi-insulating after $\mathrm{Cu}$ diffusion. The activation energies obtained from the slopes of the semi-insulating samples in both of these plots give values of 0.60 to $0.65 \mathrm{eV}$, for the samples that were initially $n$-type, and 0.70 to $0.75 \mathrm{eV}$ for the samples that were initially $p$-type. These values are close to being half the bandgap of InP. In the case when metallic precipitates play an important role in the resistivity increase, the activation energies obtained from these plots are closely related to the barrier heights in between the metallic inclusions and the host semiconductor. 


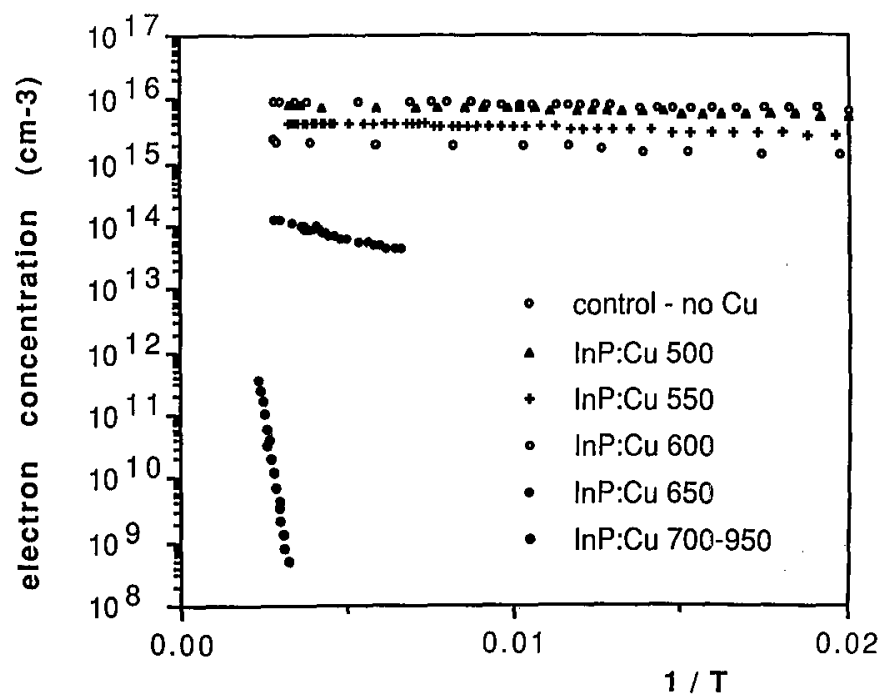

Fig. 1. Carrier concentration of $n$-InP diffused with $\mathrm{Cu}$ at different temperatures between 500 and $900^{\circ} \mathrm{C}$.

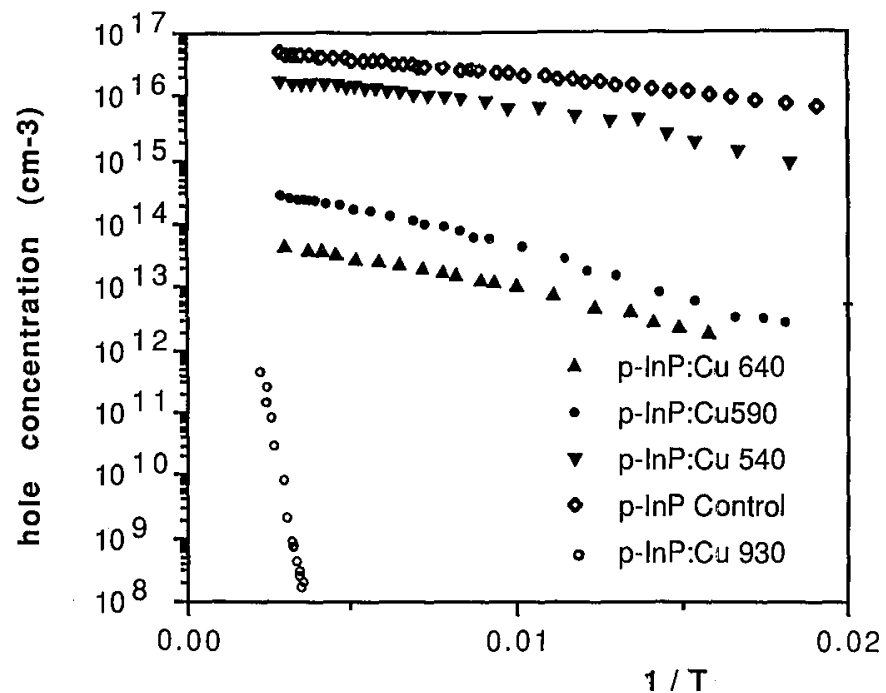

Fig. 2. Carrier concentration of $p-\operatorname{InP}: \mathrm{Cu}$ diffused at temperatures between 540 and $930^{\circ} \mathrm{C}$.

Therefore, they are not always expected to be near the middle of the gap, as the Fermi level pinning position varies with different metal/semiconductor interface.

Further observations arising from electrical measurement include a large reduction in both electron and hole mobilities which occurs mainly when the concen- 
tration of metallic precipitates is large enough to produce a significant reduction in carrier concentration but not enough to make the material semi-insulating. This sharp decrease in the mobility has been observed in InP:Cu, GaAs:Li [8] and fast neutron irradiated $\mathrm{GaAs}$ [9]. In these transitional samples for the InP:Cu study, the peak electron mobility at $70 \mathrm{~K}$ has been seen to drop from near $18,000 \mathrm{~cm}^{2} / \mathrm{V} \mathrm{s}$ in as grown nominally undoped material to $700 \mathrm{~cm}^{2} / \mathrm{V}$ s in InP:Cu diffused at $650^{\circ} \mathrm{C}$. Figure 3 shows the temperature dependence of electron mobility after different temperature diffusions in this material. The hole mobilities also show a

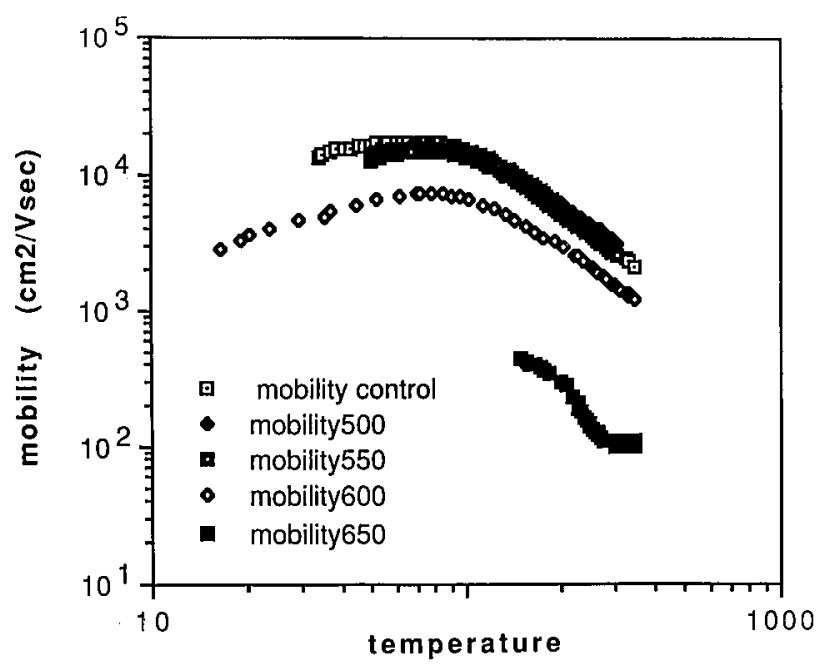

Fig. 3. Carrier mobility of $n$-InP diffused with $\mathrm{Cu}$ at temperatures between 500 and $650^{\circ} \mathrm{C}$.

drastic reduction in initially $p$-InP samples. The extremely low mobilities resulting from the diffusion of $\mathrm{Cu}$ in InP cannot be explained with scattering by ionized impurities, since a concentration greater than $10^{20} \mathrm{~cm}^{-3}$ ionized impurities would be necessary to explain the observed reduction in mobility. In these samples carrier scattering is possibly dominated by the electric field from the space charge regions, which could have an extremely large scattering cross-section when compared with the scattering cross-section for ionized impurities. The effect of metallic clusters on carrier mobility in semiconductor has been described theoretically by McNichols and Berg [9] who also showed good experimental agreement in fast neutron irradiated GaAs. Their observation of a significant mobility decrease when about one tenth of the sample volume is occupied by metallic inclusions and their associated depletion volumes is also consistent with our observations. The mobility component due to scattering of electrons from space charge regions can be expressed as [9]:

$$
\frac{1}{\mu_{\mathrm{s}}}=\left(2 m^{*} k T\right)^{1 / 2} \frac{N_{\mathrm{s}} \pi r_{\mathrm{eff}}^{2}}{q},
$$

where $m^{*}$ is the electron effective mass, $q-$ the electronic charge, $N_{\mathrm{s}}-$ the 
concentration of metallic inclusions and $r_{\mathrm{eff}}$ is the effective scattering radius for electrons by the space charge regions surrounding the precipitates. The expression for hole mobility scattering is analogous. This expression fits the mobility data obtained from Hall measurements in InP:Cu. This mobility component can also be expressed as a function of the differences in Fermi energy between the metal and semiconductors. The dependence in this case is $1 / \mu_{\mathrm{s}} \sim\left(E_{\mathrm{fs}}-E_{\mathrm{fm}}\right)^{2 / 3}$. In our observations this dependence is seen at least qualitatively, since when InP:Cu becomes semi-insulating, the room temperature mobilities seem to partially recover to what they were in the original undoped samples, before any $\mathrm{Cu}$ was introduced. In semi-insulating samples there is enough space charge region overlap to significantly flatten out $\left(E_{\mathrm{fs}}-E_{\mathrm{fm}}\right)$ reducing significantly the electrostatic potential difference responsible for scattering from space charge regions.

Other anomalous transport phenomena supporting the model for an electrically inhomogeneous material where pockets of highly conductive material are intermixed with regions of very high resistivity have been described elsewhere [5].

The presence and effectiveness of metallic precipitates will depend on a number of different factors. Naturally, there is a minimum distance in between precipitates that should be satisfied for this to occur. The depletion layer width is a function of the initial carrier concentration in the semiconductor and the Schottky barrier height, in the one-dimensional approximation for a parallel plate capacitor,

$$
w=\sqrt{\frac{2 \varepsilon \varepsilon_{0} \varphi_{\mathrm{b}}}{N_{\mathrm{A}}-N_{\mathrm{D}}}},
$$

where $\varphi_{\mathrm{b}}$ is the barrier height, $N_{\mathrm{A}}$ is the shallow acceptor concentration and $\varepsilon$ is the dielectric constant. For highly doped material, it would then be necessary to have a large concentration of metallic precipitates to obtain the required overlap that would render the material semi-insulating, whereas in lightly doped semiconductors, such as nominally undoped InP, space charge regions can be of the order of $1 / 2 \mu \mathrm{m}$, and a lower concentration of precipitates would produce the same effect. It is thus necessary to find an impurity with a large solubility at high temperatures, which also precipitates into a metallic phase upon cooling. In InP approximately $1 \times 10^{18} \mathrm{Cu}$ atoms $/ \mathrm{cm}^{3}$ and rapid quenching of the samples after diffusion are needed to create enough metallic inclusions to render the material semi-insulating. Calculations together with PIXE/Channeling and transmission electron microscopy (TEM) results show that this concentration of $\mathrm{Cu}$ gives a density of precipitates in the mid $10^{14}$ to $1 \times 10^{15}$ range, and this is sufficient to convert nominally undoped $n$-type $\mathrm{InP}$ into semi-insulating material.

The thermal stability of the metallic precipitates is also of significant concern. In the case of InP:Cu the thermal stability of the semi-insulating behavior was determined by annealing semi-insulating samples at high temperatures in sealed ampules with a weighed amount of pure $P$. The samples were annealed at temperatures of $600^{\circ} \mathrm{C}, 700^{\circ} \mathrm{C}$ and $800^{\circ} \mathrm{C}$ for $8.5,5.25$ and 3.5 hours respectively. Hall measurements of carrier concentration showed that the samples maintained a large room temperature resistivity with only a small increase in carrier concentration with respect to the unannealed semi-insulating samples. TEM examination of these samples showed that $\mathrm{Cu}-\mathrm{In}$ precipitates remain in the sample in large abundance, even after annealing at $800^{\circ} \mathrm{C}$. The semi-insulating behavior of InP:Cu thus seems 
to be stable even after high-temperature thermal treatments [6]. A good indicator of the thermal stability of precipitates can be found in the equilibrium phase diagram for the compound in question. In the case of $\mathrm{Cu}-\mathrm{In}[10]$ it is seen that these compounds are stable for a wide range of temperatures. The low substitutional solubility of $\mathrm{Cu}$ in $\mathrm{InP}$ might give the further advantage of minimizing $\mathrm{Cu}$ diffusion into the active parts of the device. The small increase in carrier concentration observed in SI samples after annealing might be due to the expected behavior of $\mathrm{Cu}-\mathrm{In}$ precipitates in the semiconductor. When the samples are heated to high temperatures, the precipitates present would partially dissolve, until the solubility of $\mathrm{Cu}$ at that temperature is reached. If all precipitates were exactly the same size, then the number of precipitates in the sample would remain consiant, as long as the annealing temperature is smaller than the original diffusion temperature. If the precipitates vary in size, which is the experimentally observed case, then some of the smaller precipitates would be completely dissolved. Upon quenching, the undissolved remaining precipitates would act as seeds for nucleation of the interstitially dissolved $\mathrm{Cu}$, and these would grow in size, with the end result of a decrease in the concentration of precipitates that existed in the sample before annealing. This would make the average distances between precipitates larger, with the consequent increase in carrier concentration. This means that one would expect better thermal stability in homogeneous samples, free of dislocations and other heterogeneous nucleation sites for precipitates.

These results show promise for obtaining a new type of semi-insulating materials. The applicability of the buried Schottky barrier model to InP:Cu indicates a general mechanism with the possibility of developing a novel type of semi-insulating semiconductors as long as a large concentration of metallic precipitates can be formed.

\section{References}

[1] A. Warren, J. Woodall, J. Freeouf, D. Grischkowsky, M. Melloch, N. Otsuka, Appl. Phys. Lett. 57, 1331 (1990).

[2] M. Kaminiska, E.R. Weber, F.W. Smith, R.A.R. Calawa, K.-M. Yu, R. Lcon, T. George, to be published.

[3] Z. Liliental-Weber, A. Claverie, J. Washbum, F. Smith, A. Calawa, Appl. Phys. A 53, 141 (1991).

[4] R.P. Leon, M. Kaminiska, K.-M. Yu, Z. Liliental-Weber, Proc. 16lh Int. Conf. Def. Semicond., Bethlehem (USA) 1991.

[5] R. Leon, M. Kamińska, K.M. Yu, E.R. Weber, to be published.

[6] R. Leon, P. Werner, C. Eder, E.R. Weber, to be published.

[7] B. Goldstein, S. Perlman, Phys. Rev. B 148, 715 (1966).

[8] H.P. Gislason, B.H. Yang, M. Linnarsson, Semi-Insulating III-V Materials, Conf. Proc. Ixtapa, 1992.

[9] J. McNichols, N. Berg, Nucl. Sci. Trans. 18, 21 (1972).

[10] J. Reynolds, W.A. Wiseman, W. Humerothery, J. Inst. Metal. 80, 637 (1980). 